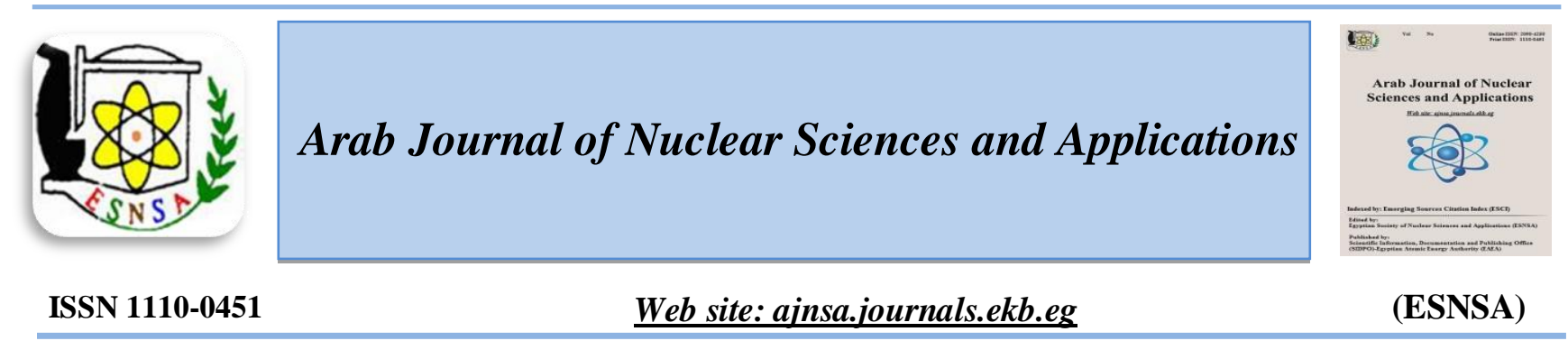

\title{
Effect of Gold Nanoparticles on the Physical Properties of Poly (3, 4- ethylenendioxythiophene): Poly (styrene sulphonate) and its Gas Sensor Application
}

\author{
Rania M. Ahmed \\ Physics Department, Faculty of Science, Zagazig University, Egypt
}

Received $17^{\text {th }}$ Dec.2017 Accepted $19^{\text {th }}$ Feb.2018

\begin{abstract}
Conducting polymers offer a unique combination of properties that make them promising candidate materials for electronic applications. Poly (3,4-ethylene dioxythiophene): poly(styrene sulphonate) (PEDOT-PSS) is one of conducting polymers that has excellent qualities to be incorporated into many organic devices as helping in the charge transfer. In this study, different sizes of gold nanosphers (Au NSs) have been embedded into the PEDOT-PSS to improve its physical properties by forming nanocomposites. Au NSs, which exhibit tunable absorption depending on their size, have effectively improved the absorbance of PEDOT-PSS. The energy gap and the Urbach energy of PEDOT-PSS have decreased from $3.45 \mathrm{eV}$ and $2566 \mathrm{meV}$, respectively, to be $3.36 \mathrm{eV}$ and $2262 \mathrm{meV}$, respectively, for the biggest size of (Au NSs) embedded in PEDOT-PSS. Also, the absorption edge value of the nanocomposite with the biggest size was $3.05 \mathrm{eV}$ whereas PEDOT-PSS has the value of $3.46 \mathrm{eV}$. Embedding Au NSs in PEDOT-PSS has caused an enhancement of the electrical conductivity of the produced nanocomposites. Lowering the gas sensitivity of PEDOT-PSS to volatile organic compound (VOCs) by embedding it with (Au NSs) can suggest this nanocomposite to be applied in electronic devices.
\end{abstract}

Keywords: Gold Nanoparticle, PEDOT-PSS, Gas Sensor, Optical Properties, Electrical Conductivity.

\section{Introduction}

Conducting polymers (CPs) have been the subject of a great deal of research due to their wide range of functional potential which makes them candidate materials for the use of in optoelectronic devices and sensors [1]. In contrast to metallic films, CPs are non-corrosive of low cost and of lightweight. They also have excellent physical and chemical properties as reasonably high electrical conductivity, ease of synthesis and design flexibility [2].

Among all CPs, poly (3, 4ethylenedioxythiophene) : poly(styrene sulfonate) (PEDOT-PSS) has attracted great attraction due to its advantages of high electrical conductivity, its high transparency in the visible range, thermal stability, and excellent atmospheric stability [3]. Moreover, PEDOT:PSS has been positioned as the best possible nominee in all organic and/or polymeric devices, electrode in light-emitting diodes, photovoltaics, memories and as sensing materials [4]. The development of PEDOT:PSS nanocomposites by carbon nanotubes, indium tin oxide nanoparticles and especially metal nanoparticles proved to be the most promising method for improving the physical properties of PEDOT:PSS such as electrical conductivity, hole transport ability and optical properties, and in consequence, its performance in optoelectronic devices [3].

The presence of Volatile Organic Compounds (VOCs) in indoor air comes at the forefront of the

Corresponding author: rania.7.8.2016@ gmail.com

DOI: 10.21608/ajnsa.2018.2326.1024

(C) Scientific Information, Documentation and Publishing Office (SIDPO)-EAEA 
imperative information that is required for the environment of human living. VOCs are emitted from extensive collection of some products like paints, cosmetic products, cleaning supplies, pesticides, organic solvents, building materials and furnishings. VOCs also comes out of the office equipment including photocopiers, printers, correction fluids, permanent markers, graphics, materials of glues and adhesives, photographic solutions [5] and some other activities such as smoking, arts, crafts and cooking [6]. Likewise, humidity levels, high temperature, strong insulation and reduced ventilation could affect many indoor environments to be as concentrators of emissions of these VOCs [5].

The conducting polymer and its nanocomposites are so sensitive toward a lower limit of (VOCs) gases in the range of a few tens of parts per million, operated close to room temperature. The interaction of conducting polymers with humidity and VOCs gases inevitably result in changes of their conductivity due to change in the interparticle distance [7]. In this context, the present study has succeeded in modifying the properties of PEDOT-PSS by embedding it with different sizes of Au NSs. To achieve this purpose, TEM, EDAX, UV-Vis spectroscopy and two points probe method have been used as effective tools to examine the different relevant properties considered. Thereafter, the obtained experimental results made it possible to test the sensitivity of PEDOT-PSS for Isopropanol gas as a VOCs gas and to study the effect of embedding PEDOT-PSS with gold nanosphere on this sensitivity.

\section{Experimental}

\section{Materials}

Orgacon Dry (Batch A650000AD) was used in this study. In addition, $\mathrm{HAuCl}_{4}$ and sodium citrate tribasic dehydrate were purchased from Sigma Aldrich Co (Germany). All chemicals were used as received and deionized water was used as a common solvent in all preparations.

\section{Preparation of the samples}

\section{Preparation of gold nanosphere (Au NSs)}

Sodium citrate reduction method as reported by Wang [8] was used to prepare gold nanosphere (Au NSs). The preparation started by heating 100 ml $\mathrm{HAuCl}_{4}$ solution $(0.01 \%)$ up to $100^{\circ} \mathrm{C}$ with stirring. Sodium citrate solutions of $1,0.750$ and
$0.691 \mathrm{ml} 1 \%$ were added to obtain different sizes of $\mathrm{Au} \mathrm{NSs}$ with stirring for $10 \mathrm{~min}$. Afterwards, the stirring process was continued for another 15 min with no heating to be cooled down to the ambient temperature. The produced gold colloid solution was kept in dark bottles at $4^{\circ} \mathrm{C}$.

\section{Preparation of nanocomposites}

An aqueous solution of PEDOT: PSS (0.005 gm in $0.15 \mathrm{ml}$ deionized water) was mixed with an aqueous suspension of $\mathrm{Au}$ NSs $(2.85 \mathrm{ml})$ from each of the different sizes to form $\mathrm{Au}$ NSs/PEDOT-PSS nanocomposites labeled by $\mathrm{C} 1$, $\mathrm{C} 2$ and $\mathrm{C} 3$ from the lowest to the largest size of $\mathrm{Au}$ NSs. The mixture was stirred for about $75 \mathrm{~min}$ to be more homogenous before casting on glass substrates $\left(2 \times 2 \mathrm{~cm}^{2}\right)$ which were cleaned previously by acetone and then deionized water in ultrasonic bath at room temperature for 15 minute for each one. The dried samples were put into a vacuum oven for $60 \mathrm{~min}$ at $80^{\circ} \mathrm{C}$ to verify the complete evaporation of the residual solvent. The thickness of the samples was measured using a stylus profilometer (DEKTAK 8000), for the casted films on the glass substrate which were approximately $1150 \mathrm{~nm}$.

\section{TEM measurement}

For understanding the morphology and the structure of the prepared samples, the transmission-electron microscopic (TEM) measurements were performed by using "TECNAI $\mathrm{F} 3 \mathrm{G}^{2 \prime}$ at $300 \mathrm{kV}$ equipped with an $\mathrm{EDAX}^{\circledR}$ system.

\section{Optical measurements}

The optical properties were studied by UV-vis/IR spectrometer (Lambda 900) in a range of wavelengths from $300 \mathrm{~nm}$ to $800 \mathrm{~nm}$.

\section{Electrical measurements}

A gold contact electrode was deposited on the surface of the casted film by using sputter coater (SCD 050, Balzers) through a shadow mask for 120 seconds with an electric current of $30 \mathrm{~mA}$ and an operating pressure of $3.6 \times 10^{-2} \mathrm{mBar}$. A little amount of silver past was dropped on the gold contact electrode to prevent scratches during the process of the measurements. Two point probe method was used for the electrical characterization using pico ampere meter (model Keithley 2400) in a 
range of applied voltages ( 0 to 7 volts) at room temperature.

\section{Gas sensor measurement}

The sensitivity of the pristine PEDOT-PSS and its nanocomposite (Sample C3) were tested to VOC of Isopropanol.

\section{Results and Discussion \\ Characterization measurements}

The prepared $\mathrm{Au}$ particles suspended in their aqueous solutions were proved to be in different nanosizes $(40-80 \mathrm{~nm})$ depending on the sodium citrate content in the preparation process as reported in a previous published work of the author [9]. Figure1 illustrates a TEM micrograph for the nanocomposites (sample C3) in which Au NSs suffer from aggregations and they are distributed densely and spaced by small distances. This aggregation can be a result of the cohesive energy of metals which is higher than that of the hosting polymer, and hence, this results in a weak interaction between metals and the polymer compared with the metal-metal binding forces [10]. It is also assumed that Au cations, during the sodium citrate reduction method, readily interact with citrate to form complexes. These complexes are transformed slower than the cations i.e. the uncomplexed ions and such slow transformation can lead to clustering of Au NSs as reported by Pillai and Kamat [11]. Eventually, the formed clusters of the Au NSs in the growth solution hamper their subsequent uniform dispersion in the polymer host [12]. The insert of Figure1 implies crystallinity of the nanocomposite due to the continuous rings of the electron diffraction patterns. EDAX analyses, as seen in Figure2, proved that the nanoparticles are Au. In addition, the presence of sulphur or sulphur oxide is surely ascribed to PEDOT-PSS [13]. The peaks of $\mathrm{Cu}$ and $\mathrm{C}$ are originated from the used TEM grid.

\section{Optical properties}

A convenient way of tuning and tailoring the optical properties of conducting polymers could be done by embedding Au NSs in the host polymer, PEDOT-PSS to form $\mathrm{Au}$ NS/PEDOT:PSS nanocomposites. In general, the visible optical absorption spectroscopy may be used as an appropriate means for verifying the formation of the gold nanoparticles. In fact, noble metal nanoparticles show a strong UV-visible absorption

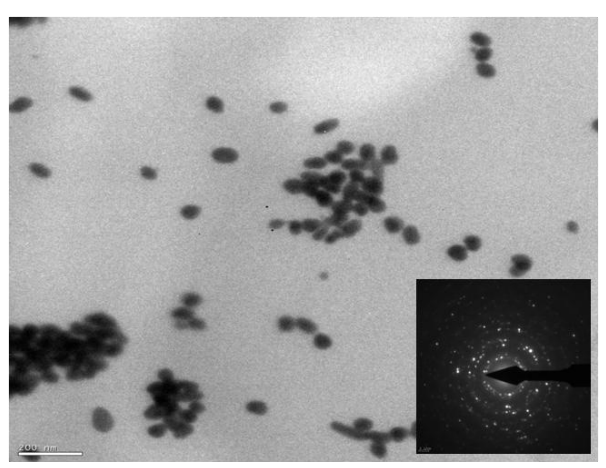

Figure 1. TEM micrographs showing the dispersion mode of the Au nanoparticle embedded in PEDOT:PSS, (the inset is about the SAED pattern of the Au NSs, synthesized via the chemical reduction method, as dispersed in deionized water)

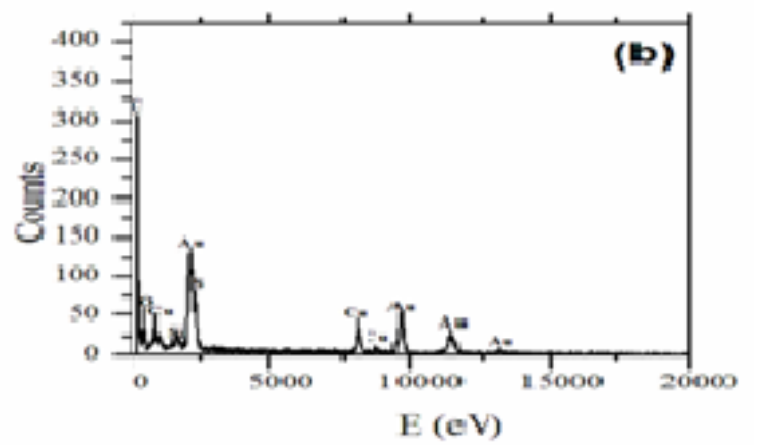

Figure 2. EDX analysis of Au NS/PEDOT:PSS

band obtained when the incident photon frequency is resonant with the collective excitation of the conduction electrons which is known as localized surface plasmon resonance (LSPR). Consequently, there will be strong surface plasmon absorption and therefore a resonance absorption peak as seen in Figure3 [14]. The appearance of a single absorption peak can be related to the transverse surface plasmon resonance of gold nanoparticles and also it gives an indication of formation spherical gold nanoparticles (Au NSs) [15]. A red shift in the wavelength of absorption maximum (524.03, 542.51, $544.82 \mathrm{~nm})$ was observed as an indication of a change in the color and the size from the smallest (red color, $1 \mathrm{ml} \mathrm{1 \%}$ sodium citrate solutions) to the largest sizes $(0.691 \mathrm{ml} 1 \%$ sodium citrate solutions dark and light purple color) of the formed Au NSs [16], see Figure3 (inset).

Studying the optical properties of a material is a good means to know the nature of its electronic structure. When a photon with enough energy has the ability to excite electrons to the highest energy from the lowest energy, the absorption process can occur. Figure 4(a) shows the absorption coefficient 
of PEDOT- PSS and its nanocomposites which were measured over a wide range of wavelengths from $300 \mathrm{~nm}$ to $800 \mathrm{~nm}$ at room temperature.

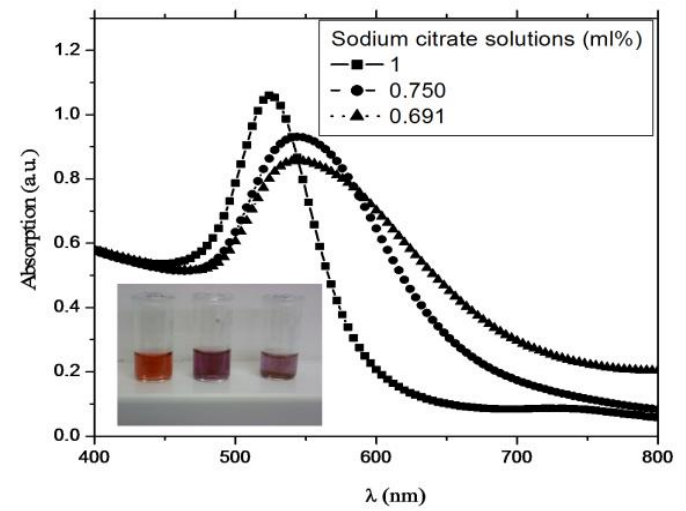

Figure 3. The UV-vis spectra of Au nanoparticle in their aqueous solutions (the inset is their colors from left (the smallest) to right (the largest)

Studying the optical properties of a material is a good means to know the nature of its electronic structure. When a photon with enough energy has the ability to excite electrons to the highest energy from the lowest energy, the absorption process can occur. Figure4 (a) shows the absorption coefficient of PEDOT- PSS and its nanocomposites which were measured over a wide range of wavelengths from $300 \mathrm{~nm}$ to $800 \mathrm{~nm}$ at room temperature.

It can be seen that the absorption coefficient of PEDOT-PSS has increased after embedding it with
$\mathrm{Au}$ NSs depending on their sizes. Figure4(b) can clarify this behavior in which dividing the absorption coefficient $(\alpha)$ of the nanocomposites films of $\mathrm{C} 1, \mathrm{C} 2$ and $\mathrm{C} 3$ by that of PEDOT-PSS film shows absorption peak positions at wavelengths of 538.6, 557.15 and $549.7 \mathrm{~nm}$, respectively. These peaks of nanocomposites films are nearly close to the corresponding peaks of the different sized $\mathrm{Au}$ NSs through their respective aqueous solutions. So, the increase in the absorption coefficient of PEDOT-PSS by embedding with $\mathrm{Au}$ NSs can be ascribed to the plasmonic effect and that is in agreement with a literature [15].

Besides, plotting the absorption coefficient, $\alpha$, against photon energy, E, illustrates that the absorption coefficient exhibits a steep rise near the absorption edge and also a straight line relationship that can be observed in the high $\alpha$ region as seen in Figure5. The intercept of the extrapolation of the linear portion of the absorption coefficient, $\alpha$, vs. photon energy, $\mathrm{E}$ to zero absorption was investigated to obtain the values of the absorption edge, $E_{e}$. The tabulated values of $E_{e}$, in Table 1 show clearly a decrease in their values with increasing $\mathrm{Au}$ NPs size embedded in PEDOT-PSS which help in understanding the band structure of the studied samples [17].
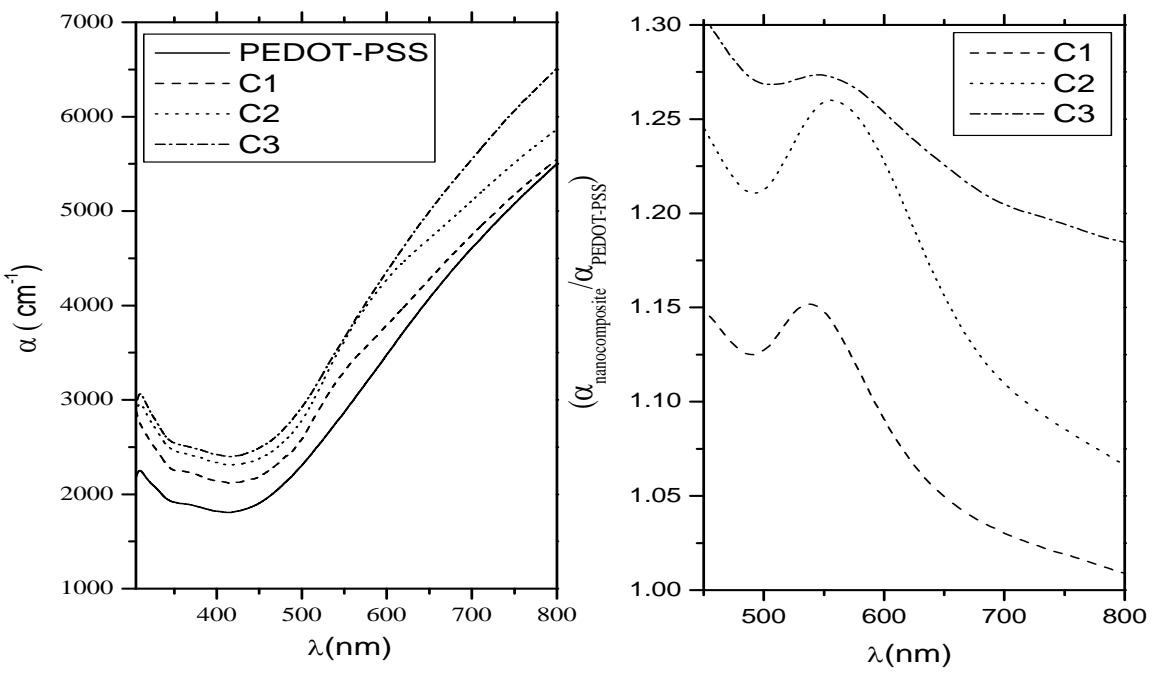

Figure 4. a) Absorption coefficient of pristine PEDOT:PSS and Au NS/PEDOT:PSS nanocomposites; and b) The absorption coefficient of $\mathrm{C} 1, \mathrm{C} 2$ and $\mathrm{C} 3$ divided by the absorption coefficient of PEDOT:PSS vs. wavelength 
Moreover, the optical energy gaps of the samples have been determined by applying the classical Tauc's expression that relates the absorption coefficient with the optical band gap. The energy band gab is determined by transforming the spectra into Tauc's plots using the energy - dependent absorption coefficient as given by Mott and Devis [18]:

$$
\alpha \mathrm{E}=\mathrm{B}\left(\mathrm{E}-\mathrm{E}_{\mathrm{g}}\right)^{\mathrm{m}}
$$

where, B is an energy-independent constant, E (hv) is the energy, $\mathrm{E}_{\mathrm{g}}$ is the energy gap and $m$ is an index having the values of $2,3,1 / 2$, and $3 / 2$, depending on the nature of electronic transition responsible for the absorption. By taking natural logarithm and derivation, we can rearrange Eq. (1) as [19]:

$$
\frac{d \ln (\alpha E)}{d E}=\frac{m}{E-E_{g}}
$$

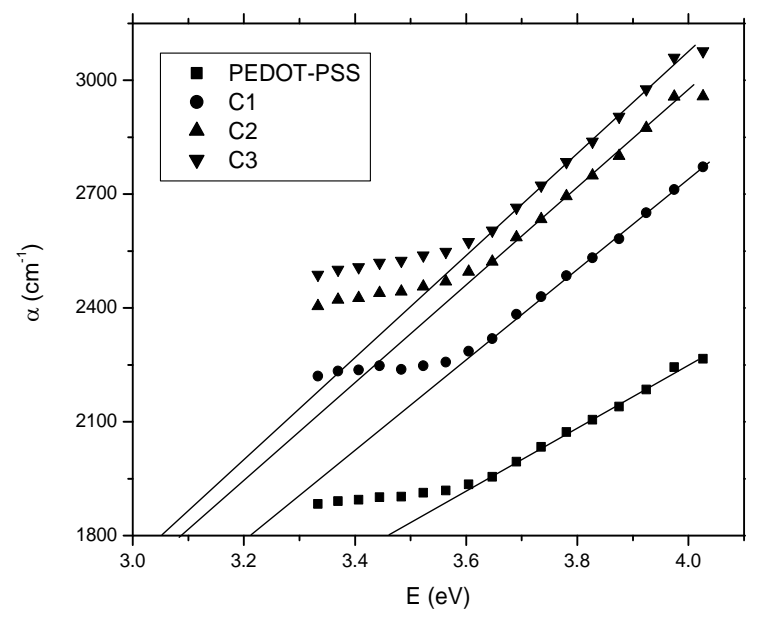

Figure 5. Absorption coefficient verses photon energy dependent for the pristine PEDOT:PSS and Au nanoparticle/PEDOT:PSS nanocomposites

Equation 3 illustrates that a peak in the curve of $\mathrm{d} \ln (\alpha \mathrm{E}) / \mathrm{dE}$ versus $\mathrm{E}$ is supposed to be observed at an energy nearly equals to $\mathrm{E}_{\mathrm{g}}$ as seen in Figure6 (a) for sample C3, as an example. This peak at a particular energy value is described as an initial value of energy gap, $\mathrm{E}_{\text {initial }}$. For all the samples, the values of $\mathrm{E}_{\text {initial }}$ were determined and listed in Table 1 to be used in plotting of $\ln (\alpha E)$ versus $\ln$ $\left(E-E_{g}\right)$ to find out the suitable value of the electronic transition, $m$ determined from the slope as shown in Figure6(b). The values of $m$, for PEDOT-PSS and its nanocomposites were in the order of 0.5 for the all. This value of $m$ can give an indication that the predominant electronic transition is the direct allowed transition.
For more accuracy, the values of the optical direct energy gap were deduced from Equation 1 by plotting $(\alpha \mathrm{E})^{2}$ against energy, $\mathrm{E}$ as seen in Figure 7 (a). The observed linear portion of this plot is an evidence that the electronic transition is direct allowed transition and consequently the optical gap, $E_{g}$, can be estimated from the intercept on the energy axis of the linear fit of the large energy data of the plot [20]. These values of optical energy gap, $\mathrm{E}_{\mathrm{g}}$, as seen in Table 1 , show a decrease in the energy gap of the pristine PEDOT-PSS by embedding it with $\mathrm{Au}$ NSs due to the formation of defects in polymeric matrix which produce the localized states in optical band gap [21].

It has been reported [22] that PEDOT-PSS which consists of disordered phases beside its ordered phases can induce the transition between both the valence band extended states and the conduction band localized states. The spreading energy of the corresponding state densities in the band gap is known as Urbach energy, $E_{u}$, which is responsible of containing a tail on the absorption spectrum known as Urbach tail. The following empirical relation of Urbach is used to determine the values of $E_{u}$ as the following [23]:

$$
\alpha=\beta \exp \left(\mathrm{E} / E_{u}\right)
$$

Where $\beta \mathrm{a}$ positive constant and $\mathrm{Eu}$ is Urbach energy or band tail energy which gives an indication about the width of the band tails of localized stated. The values of $\mathrm{E}_{\mathrm{u}}$ were estimated from the reciprocal slope of the linear portion of the plot of $\ln (\alpha)$ vs. E as seen in Fig.7(b). Table 1 shows an increase in the values of $E_{u}$ of the nanocomposites from $2197 \mathrm{meV}$, for sample $\mathrm{C} 1$, to be $2262 \mathrm{meV}$, for sample C3. This can be due to inducing disorders in PEDOT-PSS by the embedded $\mathrm{Au}$ NSs which appears in the band gap in the form of localized states depending on the size of Au NSs [24]. However, the observed low values of the Urbach energy of all nanacomposites compared to that of their hosting polymer (2566 $\mathrm{meV}$ ) may be attributed to forming clusters by incorporating Au NSs [25].

\section{Electrical Properties}

A supplementary understanding of the effect of embedding $\mathrm{Au}$ NSs into PEDOT-PSS was provided by investigating the relationship between I and V which can be expressed by [26]: 
$\mathrm{I}=\mathrm{A} \mathrm{V^{ \textrm {B } }}$

(4)
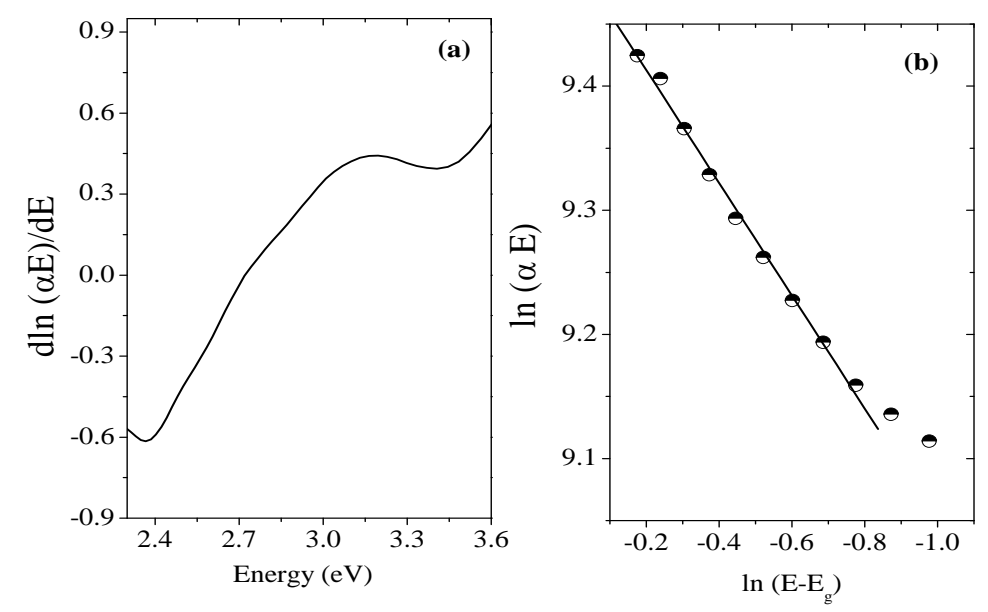

Figure 6. Plots of a) dln $(\alpha E) / d E$ versus $E$ and $b) \ln (\alpha E)$ versus $\ln \left(E-E_{g}\right)$ to determine the value of $n$ for sample $C 3$
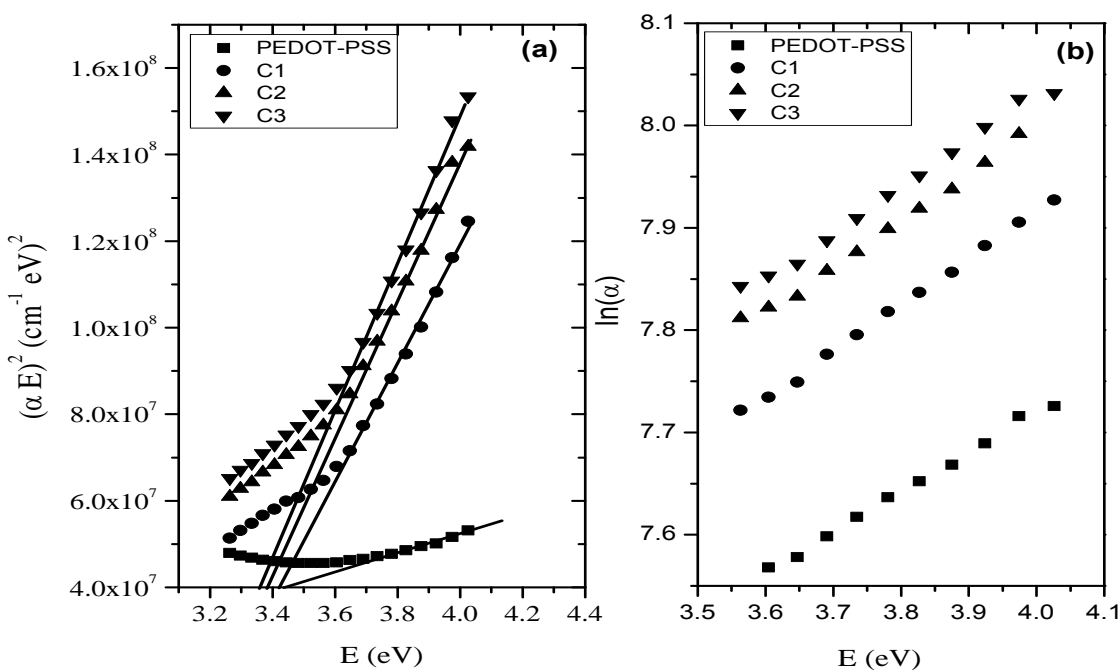

Figure 7. Dependence of $(a)(\alpha E)^{2}$ and $(b)(\ln \alpha)$ on photon energy $E(e V)$ for the pristine PEDOT:PSS and Au nanoparticle/PEDOT:PSS nanocomposites

Table 1: The values of absorption edge $\left(E_{e}\right)$, the optical band gaps $\left(E_{\text {initial }}\right.$ and $\left.E_{g}\right)$ and Urbach energy $\left(E_{u}\right)$, at room

\begin{tabular}{ccccc}
\hline Sample & $\begin{array}{c}\mathbf{E}_{\mathbf{e}} \\
(\mathbf{e V})\end{array}$ & $\begin{array}{c}\mathbf{E}_{\text {initial }} \\
(\mathbf{e V})\end{array}$ & $\begin{array}{c}\mathbf{E}_{\mathbf{g d}} \\
(\mathbf{e V})\end{array}$ & $\begin{array}{c}\mathbf{E}_{\mathbf{u}} \\
(\mathbf{m e V})\end{array}$ \\
\hline PEDOT-PSS & 3.46 & 3.213 & 3.45 & 2566 \\
\hline $\mathbf{C 1}$ & 3.21 & 3.212 & 3.42 & 2197 \\
\hline $\mathbf{C 2}$ & 3.09 & 3.193 & 3.38 & 2252 \\
\hline $\mathbf{C 3}$ & 3.05 & 3.187 & 3.36 & 2262 \\
\hline
\end{tabular}


Figure 8 shows the current density-voltage characteristics for the nanocomposites of $\mathrm{Au}$ NSs/PEDOT:PSS at room temperature. The constants B and A have been calculated and tabulated in Table 2. It could be observed that the constant B approximately equals one for all the studied samples which means that they exhibit Ohmic behavior over the experimentally range of applied voltage. A further understand may this be expanded to determine the electrical conductivity using the relation [27]:

$\mathrm{J}=\sigma \mathrm{E}$

Where $\mathrm{J}$ is the current density, $\sigma$ is the electrical conductivity and $E$ is the applied electrical field. The calculated value of $\sigma$ for the pristine PEDOT-PSS was $5.8(\mathrm{~S} / \mathrm{cm})$ as reported in a previous work of the author [28] which was improved in case of $\mathrm{Au}$ NSs addition as illustrated in Table 2. PSS is an insulating material that interrupts the flux of charge carriers through the conductive PEDOT chains. Therefore the "stripping off" of the insulating PSS from the PEDOT chains and its replacement by the Au NSs forms bonds between them and PEDOT chains can result in a reduction of the effective insulation of the PEDOT:PSS particles by the PSS. Consequently, better pathways for conduction has been existed which improve the electrical conductivity [12].
Table 2: The values of the parameters $B$ and $A$ and the electrical conductivity $(\sigma)$ at room temperature

$$
\begin{array}{llll}
\text { Sample } & \sigma\left(\Omega^{-1} \mathrm{~cm}^{-1}\right) & \mathbf{B} & \log \mathbf{A}
\end{array}
$$

\begin{tabular}{llll}
\hline C1 & 10.6 & 1.012 & -3.251 \\
\hline C2 & 16.3 & 1.017 & -3.238 \\
\hline C3 & 13.1 & 1.013 & -3.284 \\
\hline
\end{tabular}

\section{Sensor application}

For the purpose of the improvement in the conductivity of the pristine PEDOT-PSS by adding the $\mathrm{Au} \mathrm{NSs,}$ it is important to expand this study to test their sensitivity for organic vapors as Isopropanol, see Figure9. The part per million concentrations $\left(C_{p p m}\right)$ of the examined VOCs was calculated at room temperature $\left(23^{\circ} \mathrm{C}\right)$ by $C_{p p m}=C \times \frac{M}{D} \times 10^{6}$

Where $C, M$ and $D$ are the concentration, the molecular weight, and the density of the VOCs, respectively. If the VOCs are supposed to behave like an ideal gas and are saturated then their concentration can be calculated using the following equation:

$p=\frac{n}{v} R T=C R T$

Where $R$ is the ideal gas constant $\left(\mathrm{R}=8314.46 \mathrm{~cm}^{3}\right.$ kpa $\mathrm{K}^{-1} \mathrm{~mol}^{-1}$ ) and $P$ is saturated vapor pressure [28].

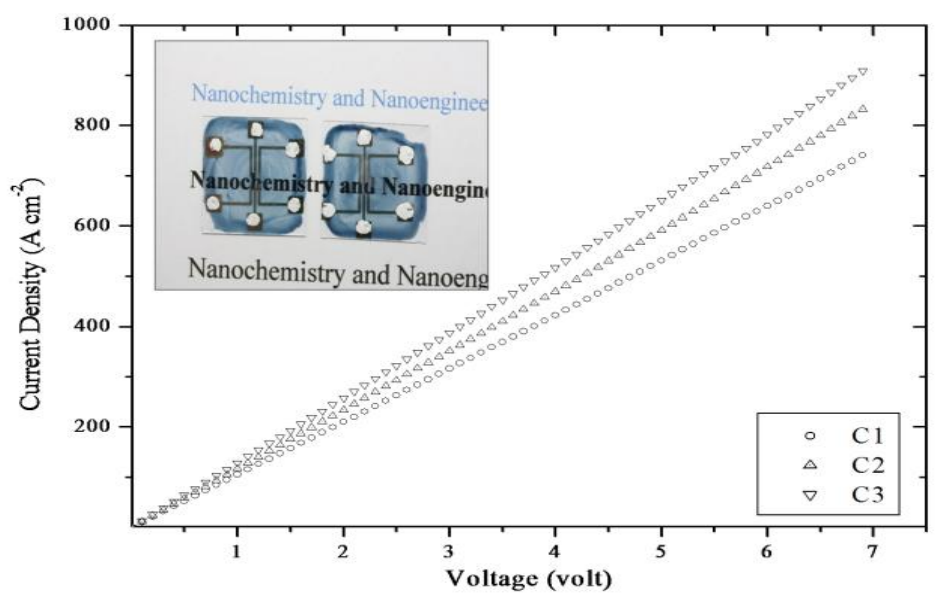

Figure 8. Current density-Voltage characteristic of PEDOT-PSS and Au NS/PEDOT:PSS nanocomposites. The inset is the photos of pure PEDOT-PSS and gold nano-sphere/PEDOT-PSS nanocomposite deposited with thin film of gold electrode, respectively from left to right 


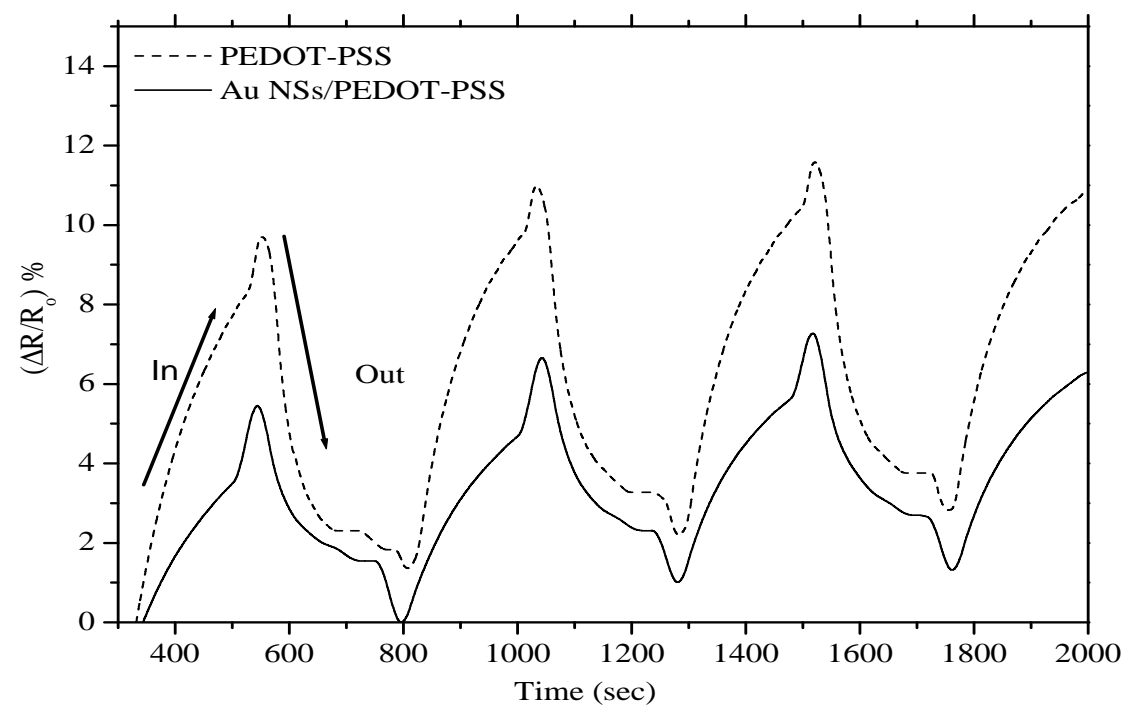

Figure 9. Resistance response of PEDOT-PSS and Au NS/PEDOT:PSS nanocomposite (sample C3) to Isopropanol vapor.

The tested Isopropanol has molar mass of 60.1 $\mathrm{g} / \mathrm{mol}$, density $(D)$ of $0.786 \mathrm{~g} / \mathrm{cm}^{3}$, molecular weight $(M)$ of $76.5 \mathrm{~cm}^{3} / \mathrm{mol}$, saturated vapor pressure $(P)$ of $5.02 \mathrm{kPa}$, and $C_{p p m}$ of 156 . The resistance change with respect to the exposure time for the VOCs was recorded under a constant applied voltage. These discerned responses were normalized to the baseline resistance measured before exposure to the VOCs. The gas sensitivity of the samples is expressed according to the following [28]:

$S \%=\frac{\Delta R}{R_{o}}=\frac{R_{f}-R_{o}}{R_{o}} \times 100$

Where $R_{f}$ is the resistance of the tested sample during exposure to the VOCs of the Isopropanol and $R_{o}$ is the resistance in air.

Because of its low molecular weight $(M=76.5$ $\mathrm{cm}^{3} / \mathrm{mol}$ ) and high part per million concentrations in air $\left(C_{p p m}=156\right)$, Isopropanol can affect the sample resistivity exposed to it. Indeed, exposing PEDOT-PSS and the nanocomposite C 3 to this organic vapor "in" shows an increasing in the resistance and vice versa when the sample is taken away from it "out". This switching of the electrical behavior could be realized as a result of swelling of the polymer by exposing to the vapor molecules and de-swelling after removing the vapor molecules. The obvious decrease in conductivity when the sample is exposed to Isopropanol gas is presumably a result of its probability of dissolving into the polymer matrix and enlarging the spacing between PEDOT chains. This behavior can affect destructively the facility of the electron hopping process and therefore leads to a reduction in the electrical conductivity value [29]. In other words, it can be said that the presence of contact points of $\mathrm{Au}$ NSs embedded in the polymer matrix have controlled the swelling process and result in lowering the high resistance that its host matrix gained during exposure to gas vapor. Therefore, $\mathrm{Au}$ NSs/ PEDOT-PSS nanocomposite has decreased the sensitivity to Isopropanol gas compared to the hosting matrix itself. Accordingly, these nanocomposites are more recommended to be applied in electronic devices than PEDOT-PSS itself.

\section{Conclusion}

TEM and EDAX are powerful tools for confirming the creation of the prepared Au NS. Embedding the pristine PEDOT-PSS with Au NSs could enhance the electrical conductivity as well as the absorption of PEDOT-PSS. The absorption spectrum showed that the electronic transition for all the studied samples was direct allowed transition in which $m$ has a value equals approximately 0.5 . The estimated value of the optical energy gap of PEDOT-PSS was decreased by embedding it with $\mathrm{Au}$ NSs. However, the values of Urbach energy for nanocomposites were less than that of the pristine PEDOT-PSS. The sensitivity of Au

NSs/PEDOT-PSS nanocomposite to gas vapor was less than that of its host polymer. This can be due to the possibility that the conductivity of PEDOT-PSS is 
negatively affected during the exposure to (VOCs) more than its nanocomposites and consequently its performance in the applied devices. Therefore, adding $\mathrm{Au}$ NSs into PEDOT-PSS gives a possibility to be applied in industry more effectively than PEDOT-PSS itself.

\section{Acknowledgement}

The author would like to express her deep gratitude to Prof. Dr. Eng. M. Elbahri for his support and allowing her preparing the samples and doing the measurements in the lab of nanochemistry and nano-engineering at CAU, Kiel, Germany, during her DAAD scholarship. The author also would like to extend her great thanks to Dr. D. Häussler and Dr. Venkata Sai Kiran Chakravadhanula, CAU, Kiel, Germany, for performing the TEM and EDAX measurements.

\section{References}

1- Abdi, M. M., Kassim, A., Ekramul Mahmud, H. N. M., Yunus, W. M. M., Talib, Z. A. and Sadrolhosseini, A. R. Physical, optical, and electrical properties of a new conducting polymer, $J$ Mater Sci. 44 (14), 3682-3686(2009).

2- Pant, H. C., Patra, M. K., Negl, S. C., Bhatia, A., Vadera, S. R. and Kumar, N. Studies on conductivity and dielectric properties of polyaniline-zinc sulphide composites, J. Bull. Mater. Sci., 29 (4), 379-384(2006).

3- Li, J., Liu, J., Gao, C., Zhang J. and Sun, H. Influence of MWCNTs Doping on the Structure and Properties of PEDOT:PSS Films, International Journal of Photoenergy, 2009, 650509(2009).

4- Choi, J., Lee, J., Choi, J., Jung, D. and Shim, S. E. Electrospun PEDOT: PSS/PVP nanofibers as the chemiresistor in chemical vapour sensing, $J$. Synthetic Metals, 160 (13-14), 1415-1421(2010).

5- Barro, R., Regueiro, J., Llompart, M. and GarciaJaresb, C. Analysis of industrial contaminants in indoor air: Part 1. Volatile organic compounds, carbonyl compounds, polycyclic aromatic hydrocarbons and polychlorinated biphenyls, Journal of Chromatography A, 1216 (3), 540 566(2009).

6- Beall, J. R. and Ulsamer, A.G. Toxicity of Volatile Organic Compounds present indoors. J. Bull N. Y. Acad. Med., 57 (10), 978-996(1981).

7- Pirsa, S. and Alizadeh, N. Design and fabrication of gas sensor based on nanostructure conductive polypyrrole for determination of volatile organic solvents J. Sensors and Actuators B, 147 (2), 461466(2010).

8- Bi, N., Sun, Y., Tian, Y., Song, D., Wang, L., Wang, J. and Zhang, H. Analysis of immunoreaction with localized surface plasmon resonance biosensor, Spectrochim. Acta Part A: Molecul. Biomolecul. Spectroscopy, 75 (3), 11631167(2010).

9- Elbahri, M., Homaeigohar, S., Abdelaziz, R., Dai, T., Khalil, R. and Zillohu, A. U Bionanocomposites: Smart Metal-Polymer Bionanocomposites as Omnidirectional Plasmonic Black Absorber Formed by Nanofluid Filtration, Adv. Funct. Mater. 22 (22), 4771-4777(2012).

10- Faupel, F. Diffusion in Non-Crystalline Metallic and Organic Media, phys. stat. sol. A, 134 (1), 959(1992).

11- Pillai, Z. S. and Kamat, P. V. What Factors Control the Size and Shape of Silver Nanoparticles in the Citrate Ion Reduction Method?, J. Phys. Chem. B, 108 (3),945-951(2004).

12- Semaltianos, N., Perrie, W., Romani, S., Potter, R., Dearden, G. and Watkins, K. Polymer-nanoparticle composites composed of PEDOT:PSS and nanoparticles of Ag synthesised by laser ablation. Colloid Polym. Sci., 290 (3), 213-220(2012).

13- Zhu, M., Eyraud, M., Rouzo, J-Le, Ahmed, N. A., Boulc'h, F., Alfonso, C., Knauth, P. and Flory, F. Simple approach for the fabrication of PEDOTcoated Si nanowires, Beilstein J. Nanotechnol, 6, 640-650(2015).

14- Raether, H. Surface plasmons on smooth and rough surfaces and on gratings, Springer Verlag, Berlin(1988).

15- Gao, H. L., Zhang, X. W., Yin, Z. G., Tan, H. R., Zhang, S. G., Meng, J. H. and Liu, X. Plasmon enhanced polymer solar cells by spin-coating $\mathrm{Au}$ nanoparticles on indium-tin-oxide substrate, Appl. Phys. Lett., 101, 133903(2012).

16- Young, J. K., Lewinski, N. A., Langsner, R. J., Kennedy, L. C., Satyanarayan, A., Nammalvar, V., Lin, A. Y. and Drezek, R. A. Size-controlled synthesis of monodispersed gold nanoparticles via carbon monoxide gas reduction", Nanoscale Res Lett., 6 (1), 428(2011).

17- Alias, A. N., Sahapini, N. F. M., Khalid, S. H. and Zabidi, Z. M. Optical Characterization of Thiophene Blending with Non-Conjugated Polymer at Different Composition, Adv Mat Res, 660, 1518(2013). 
18- Ahmed, R. M. Optical Study on Poly(methyl methacrylate)/Poly(vinyl acetate) Blends, International Journal of Photoenergy, 2009,150389(2009).

19- Muhammad, F. F., Abdul Hapip, A. I. and Sulaiman, K. Study of optoelectronic energy bands and molecular energy levels of tris (8hydroxyquinolinate) gallium and aluminum organometallic materials from their spectroscopic and electrochemical analysis, J. Organomet. Chem, 695 (23), 2526-2531(2010).

20- Marquez, E., Wagner, T., Leal, J. M., Oliva, A. M., Alcon, R. R. and Garay, R. J. Controlling the optical constants of thermally-evaporated Ge10Sb30S60 chalcogenide glass films by photodoping with silver, J. Non-Cryst. Solids, 274 (1-3), 62-68(2000).

21- Alhusiki-Alghamdi, H. M and Alghunaim, N. S. Synthesis and Characterization of Polyethylene Oxide Incorporated with Cadmium Sulphide Nanoparticles, Journal of Advances in Materials Physics and Chemistry, 5 (2), 53-59(2015).

22- Zhou, J., Anjum, D. H., Lubineau, G., Li, Er. Q. and Thoroddsen, S. T. Unraveling the order and disorder in poly (3, 4 ethylene dioxythiophene) / poly (styrenesulfonate) nanofilms, Macromolecules, 48 (16), 5688-5696(2015).

23- Ahmed, R. M. "Surface and spectroscopic properties of $\mathrm{CdSe} / \mathrm{ZnS} / \mathrm{PVC}$ nanocomposites. Polymer Composites", 38 (4), 749-758(2017).

24- Benchaabane, A., Ben Hamed, Z., Lahmar, A., Sanhoury, M. A., Kouki, F., Zellama, K., Zeinert, A. and Bouchriha, H. Optical properties of P3HT: tributylphosphine oxide-capped $\mathrm{CdSe}$ nanocomposites, Appl. Phys. A, 122 (720), 18(2016).

25- Kilarkaje, S., Manjunatha, V. and Devendrappa, H. Optical and electrical characterization of (PEO + methyl violet) polymer electrolytes, J. Appl. Polym. Sci., 124 (3), 2558-2566(2012).

26- Zhang, J., Feng, S. and Wang, X. DC current voltage characteristics of silicone rubber filled with. Conductive carbon black, J. appl. polym. sci., 94 (2), 587-592(2004).

27- Sichel, E. K. Carbn Black- Polymer Composites, Marel Dekker, INC., New York, USA(1982).

28- Khalil, R., Homaeigohar, S., Häußler, D. and Elbahri, M. A shape tailored gold-conductive polymer nanocomposite as a transparent electrode with extraordinary insensitivity to volatile organic compounds (VOCs), Sci. Rep. 6(33895), 110(2016).
29- Jian, J., Guo, X., Lin, L., Caic, Q., Cheng, J. and $\mathrm{Li}$, J. Gas-sensing characteristics of dielectrophoretically assembled composite film of oxygen plasma-treated SWCNTs and PEDOT/PSS polymer, Sensors and Actuators B, 178, 279288(2013). 